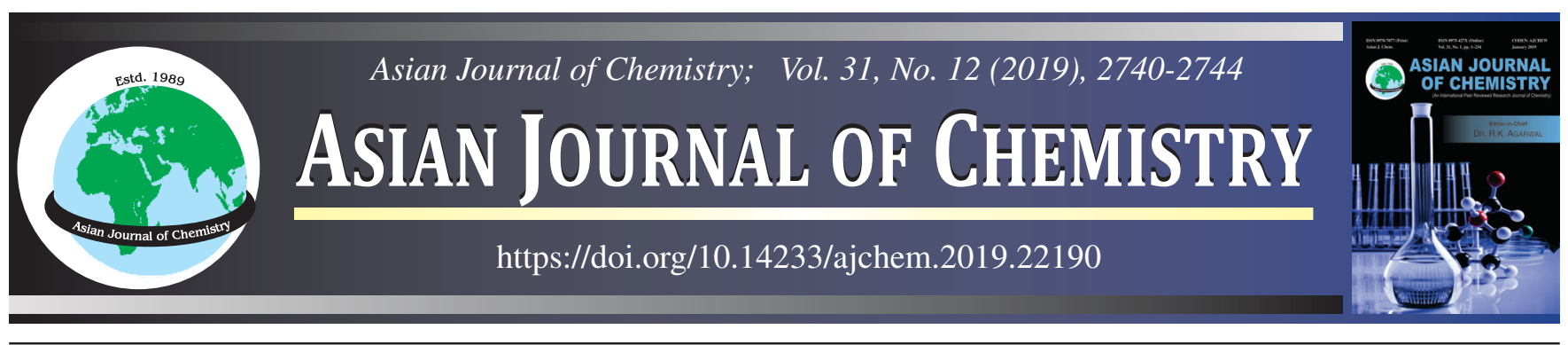

\title{
Novel Acetohydrazide Pyrazole Derivatives: Design, Synthesis, Characterization and Antimicrobial Activity
}

\author{
Anil Verma ${ }^{1}$, Vinod Kumar ${ }^{1,2}$, Ramesh Kataria $^{3}$ and Joginder Singh ${ }^{1, *}$
}

${ }^{1}$ Department of Chemistry, Maharishi Markandeshwar University (Deemed to be University), Mullana-133201, India

${ }^{2}$ Department of Chemistry, Central University of Haryana, Mahendergarh-123031, India

${ }^{3}$ Department of Chemistry, Panjab University, Chandigarh-160014, India

*Corresponding author: Fax: +91 1731 274375; Tel: +91 1731 304100; E-mail: joginderchem@mmumullana.org

Received: 10 May 2019;

Accepted: 27 June 2019;

Published online: 16 November 2019;

AJC-19613

Eleven acetohydrazide linked pyrazole derivatives were designed and synthesized via condensation of acetohyadrazide with different substituted formyl pyrazole derivatives under mild reaction conditions. Synthesized compounds were characterized on the basis of IR, NMR $\left({ }^{1} \mathrm{H} \&{ }^{13} \mathrm{C}\right)$ and mass spectrometry. The antimicrobial activities of all the compounds were screened against four bacterial and two fungal strains. Among the synthesized compounds, three compounds viz. $\mathbf{6 b}, \mathbf{6 c}$ and $\mathbf{6} \mathbf{d}$ were found as efficient antimicrobial agents in reference to the standard drugs viz. ciprofloxacin and amphotericin-B. Further, structure-activity relationship (SAR) study revealed that electron-withdrawing group enhances the antimicrobial potential of synthesized derivatives as compared to other groups present in the ring. Hence, among compounds $\mathbf{6 b}-\mathbf{c}$, compound $\mathbf{6 d}$ could be explored further against other microbes to prove its vitality.

Keywords: Acetohydrazide pyrazoles, Antimicrobial activity.

ᄂ

\section{INTRODUCTION}

The unpredictable lifestyle of the present generation and escalating resistance of microbes to presently available antimicrobial drugs, leads to numerous new diseases amongst human beings at great pace [1-3]. Different microbes such as S. aureus, B. subtilis, E. coli, $P$. aeruginosa, $C$. albicans and $S$. cerevisiae are the most frequent pathogens linked with hospital and community acquired infections [4]. Further continuous emergence of various diseases has created an unmet medical need for the development of novel antimicrobial drugs. Among various classes of antimicrobial agents, azoles possess high-quality antibacterial and antifungal potential due to their strong affinity to bind readily to enzymes and receptors in biological systems $[5,6]$.

The compounds synthesized from 2-acetylbenzofurans exhibit antimicrobial, antitumor, antiflammatory, fungicidal weed-killing activity and find use for treatment of cardiac arrhythmias [7,8]. Compounds containing pyrazole nucleus possess analgesic, antipyretic, antiarrhythmic, muscle relaxant, psychoanaleptic, anticonvulsant, hypotensive, monoamine oxidase inhibitor, antidiabetic and antibacterial activities [9-12].
Manna et al. [13] synthesized 1-acetyl-3,5-diphenyl-4,5dihydro-(1H)-pyrazole derivatives, which exhibited inhibitory activity towards amine oxidases. Introduction of phenyl ring at N1 by an acetyl group could enhance the inhibitory activity towards MAOs [13]. Yang [14] synthesized novel hydrazone derivatives having pyrazole ring by condensation of substituted aromatic aldehydes with 2-(3,5-dimethyl-1H-pyrazol1 -yl)acetohydrazide under microwave irradiation. The compounds displayed wide range of biological activities, such as anti-viral, insecticidal antitumor, analgesic, anti-inflammatory, herbicidal, antibacterial, kidney aldose reductase, etc.

In view of above mentioned facts our continuing efforts were directed to synthesize, characterize and evaluate the antifungal and antibacterial activity of pyrazole (azole) derivatives were synthesized from acetohydrazide.

\section{EXPERIMENTAL}

Melting points were measured in open glass capillary tubes with Gallenkamp melting point apparatus and are uncorrected. IR spectra of compounds were recorded on model RZX (Perkin-

This is an open access journal, and articles are distributed under the terms of the Attribution 4.0 International (CC BY 4.0) License. This license lets others distribute, remix, tweak, and build upon your work, even commercially, as long as they credit the author for the original creation. You must give appropriate credit, provide a link to the license, and indicate if changes were made. 
Elmer) spectrophotometer. The ${ }^{1} \mathrm{H}$ and ${ }^{13} \mathrm{C}$ NMR spectra were recorded on Bruker Advance DRX 400 and $100 \mathrm{MHz}$ spectrophotometer, respectively using DMSO- $d_{6}$ as a solvent. Mass spectra were recorded on UPLC-MS. The required chemicals were purchased from commercial suppliers and used without further purification. Reaction progress was monitored by thin layer chromatography using TLC sheets coated with silica gel.

Synthesis of 3-aryl-1-phenyl- $1 \mathrm{H}$-pyrazole-4-carbaldehydes (4a-k): The solution of acetophenone $(0.042 \mathrm{~mol})$ in ethanol $(10 \mathrm{~mL})$ was added to an ethanolic solution of phenylhydrazine $(0.050 \mathrm{~mol}$ in $10 \mathrm{~mL}$ EtOH$)$ at room temperature. By adding one drop of conc. $\mathrm{H}_{2} \mathrm{SO}_{4}$, reaction mixture was stirred and refluxed for $55 \mathrm{~min}$. The excess solvent was distilled off and the reaction mixture was cooled to $20-25^{\circ} \mathrm{C}$. The crude solid thus obtained was filtered, dried and recrystallized from ethanol.

To the cold solution of dimethyl formamide $(15 \mathrm{~mL})$ and acetophenone phenylhydrazone $(0.024 \mathrm{~mol})$, Vilsmeir-Haack reagent prepared from dimethylformamide $(0.071 \mathrm{~mol})$ and $\mathrm{POCl}_{3}(0.071 \mathrm{~mol})$ was added in small lots within $30 \mathrm{~min}$ at $0-5$ ${ }^{\circ} \mathrm{C}$. The reaction mixture was stirred at $60-65{ }^{\circ} \mathrm{C}$ for $5 \mathrm{~h}$ and finally poured into ice-cold water. The precipitates thus obtained on neutralization with sodium bicarbonate were filtered, washed with water and recrystallized from ethanol [15] (Scheme-I).

Further, synthesized derivative 4(a-k) and acetohydrazide (5) were refluxed in $25 \mathrm{~mL}$ ethanol for $3 \mathrm{~h}$. The reaction progress was monitored by TLC using $20 \%$ methanol + chloroform (1:4) as a solvent system. A solid thus obtained was filtered, washed and recrystallized from ethanol (Scheme-I).

1,3-Diphenyl-1 $H$-pyrazol-4-yl-methylene acetohydrazide (6a): Colour: White, yield: $92 \%$, m.p.: $170{ }^{\circ} \mathrm{C}$. Elemental analysis calcd. (found) (\%) of $\mathrm{C}_{18} \mathrm{H}_{16} \mathrm{~N}_{4} \mathrm{O}: \mathrm{C} 71.05$ (70.02); $\mathrm{H}$ 5.26 (3.29); O 5.26 (4.23); N, 18.42 (16.32). IR (KBr, $v_{\max }$ in $\left.\mathrm{cm}^{-1}\right): 1636(\mathrm{C}=\mathrm{N}$ str. $), 1704(\mathrm{C}=\mathrm{O}$ str. $) .{ }^{1} \mathrm{H}$ NMR $(400 \mathrm{MHz}$ : DMSO- $\left.d_{6}, \delta_{\mathrm{H}}, \mathrm{ppm}\right): 2.12\left(\mathrm{~s}, 3 \mathrm{H}, \mathrm{CH}_{3}\right), 11.06(\mathrm{~s}, 1 \mathrm{H}, \mathrm{NH})$, $8.23(\mathrm{~s}, 1 \mathrm{H}, \mathrm{CH}), 8.92(\mathrm{~s}, 1 \mathrm{H}$, pyrazole- $\mathrm{H}), 7.62(\mathrm{~m}, 2 \mathrm{H}, 1,5)$, 7.58 (m, 2H, 2,4), 7.45 (m, 1H, 3), 7.79 (m, 2H, 12,52), 7.51 $(\mathrm{m}, 2 \mathrm{H}, 22,42), 7.41(\mathrm{~m}, 1 \mathrm{H}, 32) .{ }^{13} \mathrm{C}$ NMR (100 MHz: DMSO$\left.d_{6}, \delta_{\mathrm{C}}, \mathrm{ppm}\right): 20.9,115.1,115.4,116.1,118.7,126.9,128.4$, $128.4,129.5,130.5,130.5,136.2,138.8,140.3,143.2,150.0$, 161.0, 163.4. Mass $(\mathrm{m} / \mathrm{z})$ : Observed $\left[\mathrm{M}^{+}\right]: 304$.

3-(4-Bromophenyl)-1-phenyl-1H-pyrazol-4-yl-methylene acetohydrazide (6b): Colour: White, yield: $89 \%$, m.p.: 154 ${ }^{\circ} \mathrm{C}$. Elemental analysis calcd. (found) (\%) of $\mathrm{C}_{18} \mathrm{H}_{15} \mathrm{~N}_{4} \mathrm{OBr}$ : C 56.39 (53.34); H 3.91 (2.95); O 4.17 (3.26); N 14.62 (13.42); $\mathrm{Br} 20.8$ (18.82). IR ( $\mathrm{KBr}, v_{\max }$ in $\left.\mathrm{cm}^{-1}\right): 1638(\mathrm{C}=\mathrm{N}$ str. $), 1705$ $\left(\mathrm{C}=\mathrm{O}\right.$ str.). ${ }^{1} \mathrm{H}$ NMR $\left(400 \mathrm{MHz}\right.$ : DMSO- $\left.d_{6}, \delta_{\mathrm{H}}, \mathrm{ppm}\right): 2.11(\mathrm{~s}$, $\left.3 \mathrm{H}, \mathrm{CH}_{3}\right), 11.08(\mathrm{~s}, 1 \mathrm{H}, \mathrm{NH}), 9.37(\mathrm{~s}, 1 \mathrm{H}, \mathrm{CH}), 9.97(\mathrm{~s}, 1 \mathrm{H}$, pyrazole- $\mathrm{H}), 7.62(\mathrm{~m}, 2 \mathrm{H}, 1,5), 7.58(\mathrm{~m}, 2 \mathrm{H}, 2,4), 7.46(\mathrm{~m}, 1 \mathrm{H}$, 3), 7.78 (m, 2H, 12,52), 7.61 (m, 2H, 22,42). ${ }^{13} \mathrm{C}$ NMR (100 MHz: DMSO- $\left.d_{6}, \delta_{\mathrm{C}}, \mathrm{ppm}\right): 21.9,116.1,116.40,118.9,126.6$, 128.6, 128.4, 129.5, 130.9, 130.6, 136.6, 138.8, 140.2, 143.0, 150.0, 158.6, 162.8, 163.5. Mass $(\mathrm{m} / \mathrm{z})$ : Observed $\left[\mathrm{M}^{+}\right]: 383$.

3-(4-Chlorophenyl)-1-phenyl-1H-pyrazol-4-yl-methylene acetohydrazide $(\mathbf{6 c})$ : Colour: White crystalline, yield: $85 \%$, m.p.: $224^{\circ} \mathrm{C}$. Elemental analysis calcd. (found) (\%) of $\mathrm{C}_{18} \mathrm{H}_{15} \mathrm{~N}_{4} \mathrm{OCl}$ : C 63.90 (61.34); $\mathrm{H} 4.43$ (3.91); O 4.73 (3.56); $\mathrm{N} 16.56$ (14.42); $\mathrm{Cl} 10.50$ (9.82). IR ( $\mathrm{KBr}, v_{\max }$ in $\left.\mathrm{cm}^{-1}\right)$ : 1639 $(\mathrm{C}=\mathrm{N} s t r),$.1706 (C=O str.). ${ }^{1} \mathrm{H}$ NMR (400 MHz: DMSO- $d_{6}$, $\left.\delta_{\mathrm{H}}, \mathrm{ppm}\right): 2.10\left(\mathrm{~s}, 3 \mathrm{H}, \mathrm{CH}_{3}\right), 11.07(\mathrm{~s}, 1 \mathrm{H}, \mathrm{NH}), 8.94(\mathrm{~s}, 1 \mathrm{H}$, $\mathrm{CH}), 9.95(\mathrm{~s}, 1 \mathrm{H}$, pyrazole-H), $7.68(\mathrm{~m}, 2 \mathrm{H}, 1,5), 7.58(\mathrm{~m}$, $2 \mathrm{H}, 2,4), 7.42(\mathrm{~m}, 1 \mathrm{H}, 3), 7.99(\mathrm{~m}, 2 \mathrm{H}, 12,52), 7.55(\mathrm{~m}, 2 \mathrm{H}$, $22,42) .{ }^{13} \mathrm{C}$ NMR $\left(100 \mathrm{MHz}\right.$ : DMSO- $\left.d_{6}, \delta_{\mathrm{C}}, \mathrm{ppm}\right): 21.5,116.9$, 118.6, 118.9, 119.2, 126.9, 127.5, 128.4, 128.5, 129.5, 129.7, 130.3, 130.8, 131.1, 135.3, 138.6, 150.2, 165.1. Mass $(\mathrm{m} / \mathrm{z})$ : Observed $\left[\mathrm{M}^{+}\right]: 338$.<smiles>[R]c1ccc(C(C)=O)cc1</smiles>

Where $=\mathrm{R}$

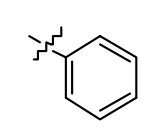

a<smiles>Cc1ccc(O)cc1</smiles>

g

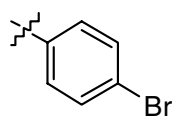

b<smiles>Cc1cccc2ccccc12</smiles>

$\mathrm{h}$<smiles>CC(C)(C)c1ccc(Cl)cc1</smiles>

c<smiles>CC(C)(C)c1ccc2ccccc2c1</smiles><smiles>Cc1ccc(F)cc1</smiles>

d<smiles>Cc1ccc(C)cc1</smiles>

e<smiles>COc1ccc(C(C)(C)C)cc1</smiles><smiles>Cc1cccs1</smiles>

j<smiles>[Y]c1c(O)cc(C)oc1=O</smiles>

Scheme-I 
TABLE-1

INHIBITION ZONE DIAMETER (mm) OF DERIVATIVES 6(a-k) AT $400 \mu \mathrm{g} / 100 \mu \mathrm{L}$ CONCENTRATION

\begin{tabular}{|c|c|c|c|c|c|c|}
\hline \multirow{2}{*}{ Compounds } & \multicolumn{2}{|c|}{ Gram-negative bacteria } & \multicolumn{2}{|c|}{ Gram-positive bacteria } & \multicolumn{2}{|c|}{ Fungi } \\
\hline & P. aeruginosa & E.coli & S. aureus & B. subtilis & S. cerevisiae & C. albicans \\
\hline 6a & 8 & 10 & 8 & 9 & 0 & 9 \\
\hline $6 \mathbf{b}$ & 9 & 12 & 12 & 10 & 12 & 14 \\
\hline $6 c$ & 10 & 14 & 12 & 12 & 14 & 16 \\
\hline 6d & 12 & 16 & 14 & 12 & 16 & 18 \\
\hline $6 e$ & 8 & 8 & 10 & 9 & 8 & 10 \\
\hline $6 f$ & 9 & 9 & 10 & 8 & 10 & 9 \\
\hline $6 \mathrm{~g}$ & 9 & 9 & 10 & 8 & 10 & 10 \\
\hline $6 h$ & 8 & 8 & 9 & 8 & 9 & 8 \\
\hline $6 \mathbf{i}$ & 9 & 10 & 8 & 9 & 8 & 9 \\
\hline $6 \mathbf{j}$ & 9 & 9 & 10 & 10 & 9 & 11 \\
\hline $6 \mathbf{k}$ & 9 & 10 & 10 & 9 & 11 & 12 \\
\hline Ciprofloxacin & 16 & 18 & 18 & 16 & - & - \\
\hline Amphotericin B & - & - & - & - & 20 & 18 \\
\hline
\end{tabular}

3-(4-Fluorophenyl)-1-phenyl-1H-pyrazol-4-yl-methylene acetohydrazide (6d): Colour: Cream colour, yield: $90 \%$, m.p.: $184{ }^{\circ} \mathrm{C}$. Elemental analysis calcd. (found) $(\%)$ of $\mathrm{C}_{18} \mathrm{H}_{15} \mathrm{~N}_{4} \mathrm{OF}$ : C 67.08 (63.34); H 4.65 (3.41); O 4.96 (3.36); N 17.39 (15.42); F 5.90 (4.81). IR (KBr, $v_{\max }$ in $\left.\mathrm{cm}^{-1}\right)$ : 1635 (C=N str.), 1707 $(\mathrm{C}=\mathrm{O}$ str. $) .{ }^{1} \mathrm{H}$ NMR $\left(400 \mathrm{MHz}\right.$ : DMSO- $\left.d_{6}, \delta_{\mathrm{H}}, \mathrm{ppm}\right): 2.10$ (s, $\left.3 \mathrm{H}, \mathrm{CH}_{3}\right), 11.32$ (s, 1H, NH), 8.34 (s, 1H, CH), $8.52(\mathrm{~s}, 1 \mathrm{H}$, pyrazole-H), $7.66(\mathrm{~m}, 2 \mathrm{H}, 1,5), 7.54(\mathrm{~m}, 2 \mathrm{H}, 2,4), 7.49$ (m, 1H, 3), 8.15 (m, 2H, 12,52), 7.32 (m, 2H, 22,42). ${ }^{13} \mathrm{C} \mathrm{NMR}(100$ MHz: DMSO- $\left.d_{6}, \delta_{\mathrm{C}}, \mathrm{ppm}\right): 21.6,116.1,116.40,118.9,126.6$, $128.6,128.4$, 129.5, 130.9, 130.6, 136.6, 138.8, 140.2, 143.0, 150.0, 158.6, 162.8, 163.5. Mass ( $\mathrm{m} / \mathrm{z})$ : Observed $\left[\mathrm{M}^{+}\right]$: 322.

1-Phenyl-3-(p-tolyl)-1H-pyrazol-4-yl-methylene acetohydrazide (6e): Colour: Cream white, yield: $76 \%$, m.p.: 212 ${ }^{\circ} \mathrm{C}$. Elemental analysis calcd. (found) $(\%)$ of $\mathrm{C}_{19} \mathrm{H}_{18} \mathrm{~N}_{4} \mathrm{O}: \mathrm{C}$ 71.69 (69.34); H 4.65 (3.49); O 5.03 (4.36); N 17.61 (16.42). IR (KBr, $v_{\max }$ in $\left.\mathrm{cm}^{-1}\right)$ : 1639 (C=N str.), 1708 (C=O str.). ${ }^{1} \mathrm{H}$ NMR (400 MHz: DMSO- $\left.d_{6}, \delta_{\mathrm{H}}, \mathrm{ppm}\right): 2.14$ (s, 3H, $\left.\mathrm{CH}_{3}\right), 11.23$ (s, 1H, NH), $9.30(\mathrm{~s}, 1 \mathrm{H}, \mathrm{CH}), 9.97(\mathrm{~s}, 1 \mathrm{H}$, pyrazole-H), 7.65 (m, 2H, 1,2), 7.57 (m, 2H, 2,4), 7.44 (m, 1H, 3), 7.67 (m, 2H, 12,52), 7.33 (m, 2H, 22,42), 2.49 (s, 3H, $\left.\mathrm{CH}_{3}\right) .{ }^{13} \mathrm{C}$ NMR (100 MHz: DMSO- $\left.d_{6}, \delta_{\mathrm{C}}, \mathrm{ppm}\right): 21.5,116.7,116.8,118.6,126.7$, 128.2, 129.0, 129.1, 129.3, 129.5, 135.7, 137.6, 137.9, 138.8, 139.2, 151.1, 151.7, 162.8, 165.1. Mass $(\mathrm{m} / \mathrm{z})$ : Observed $\left[\mathrm{M}^{+}\right]$: 318.

3-(4-Methoxyphenyl)-1-phenyl- $1 H$-pyrazol-4-ylmethylene acetohydrazide (6f): Colour: Creamy colour, yield: $79 \%$, m.p.: $190{ }^{\circ} \mathrm{C}$. Elemental analysis calcd. (found) (\%) of $\mathrm{C}_{18} \mathrm{H}_{15} \mathrm{~N}_{4} \mathrm{O}_{2}$ : C 68.26 (66.34); H 5.38 (4.49); O 9.58 (7.36); $\mathrm{N}$ 16.76 (15.42). IR ( $\mathrm{KBr}, v_{\max }$ in $\left.\mathrm{cm}^{-1}\right): 1637$ (C=N str.), 1705 $(\mathrm{C}=\mathrm{O}$ str. $) .{ }^{1} \mathrm{H}$ NMR $\left(400 \mathrm{MHz}: \mathrm{DMSO}-d_{6}, \delta_{\mathrm{H}}, \mathrm{ppm}\right): 2.14(\mathrm{~s}$, $\left.3 \mathrm{H}, \mathrm{CH}_{3}\right), 11.19$ (s, 1H, NH), 8.84 (s, 1H, CH), 9.92 (s, 1H, pyrazole-H), $7.66(\mathrm{~m}, 2 \mathrm{H}, 1,5), 7.56(\mathrm{~m}, 2 \mathrm{H}, 2,4), 7.43(\mathrm{~m}$, 1H, 3), 7.55 (m, 2H, 12,52), 7.08 (m, 2H, 22,42), 3.82 (s, 3H, $\left.\mathrm{OCH}_{3}\right) .{ }^{13} \mathrm{C}$ NMR $\left(100 \mathrm{MHz}\right.$ : DMSO- $\left.d_{6}, \delta_{\mathrm{C}}, \mathrm{ppm}\right): 21.3,116.5$, 116.6, 118.6, 126.7, 129.5, 129.6, 129.7, 130.0, 135.8, 137.6, $138.5,138.9,139.2,152.5,159.4,160.8,165.7$. Mass $(\mathrm{m} / \mathrm{z})$ : Observed $\left[\mathrm{M}^{+}\right]: 334$.

3-(4-Hydroxyphenyl)-1-phenyl-1H-pyrazol-4-ylmethylene acetohydrazide (6g): Colour: Yellowish colour, yield: $67 \%$, m.p.: $230{ }^{\circ} \mathrm{C}$. Elemental analysis calcd. (found) $\mathrm{C}_{18} \mathrm{H}_{16} \mathrm{~N}_{4} \mathrm{O}_{2}$ : C 67.50 (65.24); H 5.01 (4.19); O 10.04 (8.36); $\mathrm{N} 17.50$ (15.12). IR (KBr, $v_{\max }$ in $\left.\mathrm{cm}^{-1}\right): 1636$ (C=N str.), 1703 $(\mathrm{C}=\mathrm{O}$ str. $) .{ }^{1} \mathrm{H}$ NMR (400 MHz: DMSO- $\left.d_{6}, \delta_{\mathrm{H}}, \mathrm{ppm}\right): 2.32(\mathrm{~s}$, $\left.3 \mathrm{H}, \mathrm{CH}_{3}\right), 11.03$ (s, 1H, NH), 9.68 (s, 1H, CH), 9.86 (s, 1H, pyrazole-H), $7.62(\mathrm{~m}, 2 \mathrm{H}, 1,5), 7.58(\mathrm{~m}, 2 \mathrm{H}, 2,4), 7.45$ (m, 1H, 3), 7.49 (m, 2H, 12,52), 7.05 (m, 2H, 22,42), 5.35 (s, 1H, $\mathrm{OH}) .{ }^{13} \mathrm{C}$ NMR (100 MHz: DMSO- $\left.d_{6}, \delta_{\mathrm{C}}, \mathrm{ppm}\right): 21.7,116.5$, 116.6, 118.5, 122.7, 126.6, 129.5, 129.6, 129.7, 130.0, 136.8, $139.0,151.3,151.9,157.8,159.4,160.8,165.2$. Mass $(\mathrm{m} / \mathrm{z})$ : Observed $\left[\mathrm{M}^{+}\right]: 320$.

3-(Naphthalen-1-yl)-1-phenyl-1H-pyrazol-4-yl-methylene acetohydrazide (6h): Colour: Creamy white, yield: 71 $\%$, m.p.: $168^{\circ} \mathrm{C}$. Elemental analysis calcd. (found) $\mathrm{C}_{22} \mathrm{H}_{18} \mathrm{~N}_{4} \mathrm{O}$ : C 74.59 (72.24); H 5.08 (4.09); O 4.51 (3.36); N 15.81 (13.12). IR ( $\mathrm{KBr}, \mathrm{v}_{\max }$ in $\left.\mathrm{cm}^{-1}\right)$ : $1638(\mathrm{C}=\mathrm{N}$ str. $), 1709 \mathrm{~cm}^{-1}(\mathrm{C}=\mathrm{O}$ str. $)$. ${ }^{1} \mathrm{H}$ NMR (400 MHz: DMSO- $d_{6}, \delta_{\mathrm{H}}, \mathrm{ppm}$ ): 2.50 (s, $3 \mathrm{H}, \mathrm{CH}_{3}$ ), 11.06 (s, 1H, NH), 9.43 (s, 1H, CH), 9.73 (s, 1H, pyrazole-H), 7.64 (m, 2H, 1,5), 7.53 (m, 2H, 2,4), 7.48 (m, 1H, 3), 7.62 (m, 1H, 12), 7.58 (m, 1H, 22), 7.75 (m, 1H, 32), 7.84 (s, 1H, 42), 7.55 (s, 2H, 52,62), 7.90 (m, 1H, 72). ${ }^{13} \mathrm{C}$ NMR (100 MHz: DMSO- $\left.d_{6}, \delta_{\mathrm{C}}, \mathrm{ppm}\right): 20.9,118.5,118.7,119.3,123.5,125.3$, 125.6, 126.4, 127.7, 128.6, 129.3, 129.6, 129.7, 131.6, 133.2, $134.8,138.3,138.6,150.1,151.2,152.3,165.2$. Mass $(\mathrm{m} / \mathrm{z})$ : Observed $\left[\mathrm{M}^{+}\right]: 354$.

3-(Naphthalen-2-yl)-1-phenyl-1H-pyrazol-4-yl-methylene acetohydrazide (6i): Colour: White, yield: $68 \%$, m.p.: $152{ }^{\circ} \mathrm{C}$. Elemental analysis calcd. (found) $(\%)$ of $\mathrm{C}_{22} \mathrm{H}_{18} \mathrm{~N}_{4} \mathrm{O}$ : C 74.59 (71.24); H 5.08 (3.09); O 4.51 (3.66); N 15.81 (13.12). IR ( $\mathrm{KBr}, \mathrm{v}_{\max }$ in $\left.\mathrm{cm}^{-1}\right): 1640(\mathrm{C}=\mathrm{N}$ str. $), 1708(\mathrm{C}=\mathrm{O}$ str. $) .{ }^{1} \mathrm{H}$ NMR (400 MHz: DMSO- $\left.d_{6}, \delta_{\mathrm{H}}, \mathrm{ppm}\right): 2.50$ (s, 3H, $\left.\mathrm{CH}_{3}\right), 11.28$ (s, 1H, NH), 8.42 (s, 1H, CH), 8.98 (s, 1H, pyrazole-H), 7.68 (m, 2H, 1,5), $7.51(\mathrm{~m}, 2 \mathrm{H}, 2,4), 7.43(\mathrm{~m}, 1 \mathrm{H}, 3), 7.62(\mathrm{~m}, 1 \mathrm{H}$, 12), 8.62 (m, 1H, 22), 7.58 (s, 1H, 42,52), 7.98 (s, 1H, 32,62), $7.91(\mathrm{~m}, 1 \mathrm{H}, 72) .{ }^{13} \mathrm{C}$ NMR (100 MHz: DMSO- $\left.d_{6}, \delta_{\mathrm{C}}, \mathrm{ppm}\right)$ : 21.6, 117.2, 118.6, 118.7, 119.3, 126.2, 126.4, 126.5, 127.5, 127.9, 128.4, 129.4, 129.5, 129.7, 132.6, 132.8, 135.8, 138.7, 139.0, 150.8, 151.3, 165.2. Mass $(\mathrm{m} / \mathrm{z})$ : Observed $\left[\mathrm{M}^{+}\right]: 354$.

1-Phenyl-3-(thiophen-2-yl)-1H-pyrazol-4-yl-methylene acetohydrazide (6j): Colour: Brownish crystalline, yield: 
$65 \%$, m.p.: $232{ }^{\circ} \mathrm{C}$; Elemental analysis calcd. (found) (\%) of $\mathrm{C}_{16} \mathrm{H}_{14} \mathrm{~N}_{4} \mathrm{OS}$ : C 61.93 (59.24); H, 4.51 (3.69); O 5.16 (4.66); $\mathrm{N} 18.06$ (16.12); S 10.32 (8.32). IR (KBr, $v_{\max }$ in $\left.\mathrm{cm}^{-1}\right)$ : 1635 $(\mathrm{C}=\mathrm{N}$ str. $), 1706(\mathrm{C}=\mathrm{O}$ str. $) .{ }^{1} \mathrm{H}$ NMR (400 MHz: DMSO- $d_{6}$, $\left.\delta_{\mathrm{H}}, \mathrm{ppm}\right): 2.20\left(\mathrm{~s}, 3 \mathrm{H}, \mathrm{CH}_{3}\right), 11.33(\mathrm{~s}, 1 \mathrm{H}, \mathrm{NH}), 8.94(\mathrm{~s}, 1 \mathrm{H}$, $\mathrm{CH}), 9.37(\mathrm{~s}, 1 \mathrm{H}$, pyrazole-H), $7.69(\mathrm{~m}, 2 \mathrm{H}, 1,5), 7.52(\mathrm{~m}$, $2 \mathrm{H}, 2,4), 7.45(\mathrm{~m}, 1 \mathrm{H}, 3), 7.55(\mathrm{~m}, 1 \mathrm{H}, 12), 7.68(\mathrm{~m}, 1 \mathrm{H}, 22)$, 7.69 (m, $1 \mathrm{H}, 32) .{ }^{13} \mathrm{C}$ NMR (100 MHz: DMSO- $\left.d_{6}, \delta_{\mathrm{C}}, \mathrm{ppm}\right)$ : $21.9,116.3,118.5,119.1,126.8,126.9,127.6,127.7,128.3$, 129.5, 129.7, 133.3, 135.4, 138.6, 145.6, 165.8. Mass $(\mathrm{m} / \mathrm{z})$ : Observed $\left[\mathrm{M}^{+}\right]$: 310 .

3-(Hydroxy-4-methyl-6-oxocyclohexa-1,3-dien-1-yl)-1phenyl-1H-pyrazol-4-yl-methylene acetohydrazide $(6 \mathrm{k})$ : Colour: Yellow colour, yield: $68 \%$, m.p.: $154{ }^{\circ} \mathrm{C}$. Elemental analysis calcd. (found) (\%) of $\mathrm{C}_{18} \mathrm{H}_{16} \mathrm{~N}_{4} \mathrm{O}_{4}$ : C 61.36 (59.12); H 4.54 (3.19); O 18.16 (15.66); N 15.90 (12.12). IR (KBr, $v_{\max }$ in $\left.\mathrm{cm}^{-1}\right): 1640(\mathrm{C}=\mathrm{N}$ str. $), 1707(\mathrm{C}=\mathrm{O}$ str. $) ;{ }^{1} \mathrm{H}$ NMR $(400$ MHz: DMSO- $\left.d_{6}, \delta_{\mathrm{H}}, \mathrm{ppm}\right): 2.22\left(\mathrm{~s}, 3 \mathrm{H}, \mathrm{CH}_{3}\right), 11.02(\mathrm{~s}, 1 \mathrm{H}$, $\mathrm{NH}), 8.59(\mathrm{~s}, 1 \mathrm{H}, \mathrm{CH}), 9.84(\mathrm{~s}, 1 \mathrm{H}$, pyrazole-H), $7.62(\mathrm{~m}, 2 \mathrm{H}$, 1,5), $7.59(\mathrm{~m}, 2 \mathrm{H}, 2,4), 7.46(\mathrm{~m}, 1 \mathrm{H}, 3), 10.93(\mathrm{~s}, 1 \mathrm{H}, \mathrm{OH}), 7.23$ (s, $1 \mathrm{H}, 12), 1.82\left(\mathrm{~s}, 3 \mathrm{H}, \mathrm{CH}_{3}\right) .{ }^{13} \mathrm{C}$ NMR (100 MHz: DMSO$\left.d_{6}, \delta_{\mathrm{C}}, \mathrm{ppm}\right): 45.7,107.8,116.0,119.5,119.8,123.5,124.9$, 126.9, 126.3, 128.5, 129.5, 129.7, 139.3, 141.5, 143.6, 165.2, 171.5, 184.1. Mass $(\mathrm{m} / \mathrm{z})$ : Observed $\left[\mathbf{M}^{+}\right]: 352$.

Microorganisms: Six microbial strains viz. E. coli, S. aureus, C. albicans, $P$. aeruginosa, $B$. subtilis and $S$. cerevisiae were selected for desired activity by analyzing their clinical importance in human beings. The microbial cultures used for testing purpose were supplied by Microbial Type Culture Collection (MTCC), IMTECH, Chandigarh, India. The medium used for sub-culturing of bacterial strains as well as yeasts were nutrient agar and malt extract agar (MEA), respectively.

Zone of inhibition: Agar well diffusion technique was adopted to measure the inhibitory zone of prepared derivatives [16]. The inoculums of selected microbes were obtained from cultures matured for $16 \mathrm{~h}$ having $10^{8} \mathrm{cfu} / \mathrm{mL}$ concentrations. Twenty $\mathrm{mL}$ of nutrient agar and malt extract agar (MEA) plates were transferred separately in petri plates and swabbed with required amount of the inoculums of test microbes. The plates were left alone for about 15 min for proper adsorption. Each derivative dissolved in dimethylsulfoxide (DMSO) was loaded into wells of $8 \mathrm{~mm}$ diameters with $100 \mu \mathrm{L}$ of concentration $(4.0 \mathrm{mg} / \mathrm{mL})$. Incubation of plates was carried out for $24 \mathrm{~h}$ at 37 ${ }^{\circ} \mathrm{C}$. The anti-microbial potential of derivatives in opposition to the preferred microbes was tested by measuring inhibitory zone through zone reader. Ciprofloxacin and amphotericin-B were taken as positive control for bacterial and yeast microbes respectively. For each organism, this procedure was performed thrice.

Minimum inhibitory concentration (MIC): MIC of synthesized derivatives was obtained using modified agar well diffusion method. The synthesized derivatives dissolved in DMSO, were further diluted with distilled water to attain the desired concentration range $(4-0.0625 \mathrm{mg} / \mathrm{mL})$ [17]. The wells (triplicates) were filled with $100 \mu \mathrm{L}$ of each dilutions of derivatives and further incubated aerobically at $37^{\circ} \mathrm{C}$ for $24 \mathrm{~h}$ to study the inhibition zones. Positive and negative controls used in this investigation were ciprofloxacin, amphotericin-B and DMSO, respec- tively. Triplicates were maintained and the experiments were repeated thrice [18].

\section{RESULTS AND DISCUSSION}

Synthesis of various pyazoles viz. 1-phenyl-3-substituted phenyl-1 $H$-pyrazol-4-yl-methylene acetohydrazide derivatives were achieved (Scheme-I). The synthesized compounds were characterized by analyzing their ${ }^{1} \mathrm{H} \&{ }^{13} \mathrm{C} N M R$, IR and mass spectral data. The $-\mathrm{NH}$ and $-\mathrm{C}=\mathrm{O}$ stretching vibrations of all the derivatives of appeared near 3329 and $1674 \mathrm{~cm}^{-1}$, respectively. Two singlets due to pyrazole $-\mathrm{H}$ and $\mathrm{N}=\mathrm{CH}$ at $\delta 9.73$ and 9.43, respectively confirmed the formation of acetyl pyrazole derivatives or acetohydrazide linkage containing pyrazoles. The compounds $\mathbf{6}(\mathbf{a}-\mathbf{k})$ displayed signals in ${ }^{13} \mathrm{C}$ NMR spectra in the range of $\delta 139.02$ to $\delta 150.87$ corresponding to pyrazole ring carbons, which assured the formation of acetohydrazide pyrazole derivatives.

Antimicrobial activity: The outcomes of antimicrobial screening revealed that among all the tested compounds, compounds $\mathbf{6 c}$ and $\mathbf{6 d}$ exhibited good inhibitory potential against to all the four bacterial and two fungal microbes. The maximum diameter of inhibitory zone was $16 \mathrm{~mm}$ in bacterial strains and $18 \mathrm{~mm}$ in fungal strains shown by compound $\mathbf{6 d}$. The inhibitory potential was $89 \%, 78 \%$ against $E$. coli, $S$. aureus, $75 \%$ for $P$. aeruginosa and $B$. subtilis, respectively as compared to the standard antibacterial ciprofloxacin (Table-1). The inhibitory antifungal potential was 80 and $100 \%$ against $S$. cerevisiae, C. albicans, respectively when compared with standard amphotericin-B.

SAR of synthesized compounds 6(a-k): Among the derivatives $\mathbf{6}(\mathbf{a}-\mathbf{k})$, derivative $\mathbf{6 d}$ having fluoro phenyl group at pyrazole ring displayed the highest antimicrobial activity against all the microbes. The antimicrobial potential decreases in derivative $\mathbf{6 c}$ having chloro phenyl group at the pyrazole ring in comparison to compound $\mathbf{6 d}$.

In case of derivative $\mathbf{6 b}$ with bromo phenyl group at pyrazole ring, the antibacterial potential further decreases as compared to compounds $\mathbf{6 c}$ and $\mathbf{6 d}$ analogues. Thus, it can be concluded that electron-withdrawing groups enhance the antimicrobial potential of synthesized derivatives as compared to other groups present in the ring. Hence, compound $\mathbf{6 d}$ exhibited maximum antimicrobial potential due to high electronegativity of fluorine.

\section{Conclusion}

The synthesis of total eleven novel pyrazoles viz. (1-phenyl3 -substituted phenyl-1 $H$-pyrazol-4-yl-methylene acetohydrazide) derivatives are reported. All the synthesized derivatives were characterized by analyzing their IR, NMR $\left({ }^{1} \mathrm{H} \&{ }^{13} \mathrm{C}\right)$ and mass spectral data. The synthesized compounds were screened for their antimicrobial properties and exhibited significant potential against bacterial and fungal strains. Compound $\mathbf{6 d}$ was found to exhibit high inhibitory potential and can serve as potent antimicrobial lead for further studies.

\section{CONFLICT OF INTEREST}

The authors declare that there is no conflict of interests regarding the publication of this article. 


\section{REFERENCES}

1. R. Basawaraj, B. Yadav and S.S. Sangapure, Indian J. Heterocycl. Chem., 11, 31 (2001).

2. S.M. Rida, S.A.M. El-Hawash, H.T.Y. Fahmy, A.A. Hazzaa and M.M.M. El-Meligy, Arch. Pharm. Res., 29, 826 (2006); https://doi.org/10.1007/BF02973901.

3. R.K. Ujjinamatada, R.S. Appala and Y.S. Agasimundin, J. Heterocycl. Chem., 43, 437 (2006); https://doi.org/10.1002/jhet.5570430226.

4. S. Wachi, K. Takagi, G. Menichi and M. Hubert-Habart, Bull. Soc. Chim. Fr, 5-6, 230 (1978).

5. D.V. Singh, A.R. Mishra, R.M. Mishra, A.K. Pandey, C.R. Singh and A.K. Dwivedi, Indian J. Heterocycl. Chem., 14, 319 (2005).

6. J.U. Cho and C.J. Kim H.B. Lee and C.H. Lim, Patent Korea KR 2004064795 (2004); Chem. Abstr., 145, 182279 (2004).

7. P. Druzgala and P.G. Milner, Novel Enantiomeric Compounds for Treatment of Cardiac Arrhythmias and Methods of Use, U.S. Patent 20020193428 (2002).

8. K.M. Dawood, H. Abdel-Gawad, E.A. Rageb, M. Ellithey and H.A. Mohamed, Bioorg. Med. Chem., 14, 3672 (2006); https://doi.org/10.1016/j.bmc.2006.01.033.

9. P.D. Sauzem, P. Machado, M.A. Rubin, G. da S. Sant'Anna, H.B. Faber, A.H. de Souza, C.F. Mello, P. Beck, R.A. Burrow, H.G. Bonacorso, N. Zanatta and M.A.P. Martins, Eur. J. Med. Chem., 43, 1237 (2008); https://doi.org/10.1016/j.ejmech.2007.07.018.
10. A. Hall, A. Billinton, S.H. Brown, N.M. Clayton, A. Chowdhury, G.M.P. Giblin, P. Goldsmith, T.G. Hayhow, D.N. Hurst, I.R. Kilford, A. Naylor, B. Passingham and L. Winyard, Bioorg. Med. Chem. Lett., 18, 3392 (2008); https://doi.org/10.1016/j.bmcl.2008.04.018.

11. F.R. Souza, V.T. Souza, V. Ratzlaff, L.P. Borges, M.R. Oliveira, H.G. Bonacorso, N. Zanatta, M.A.P. Martins and C.F. Mello, Eur. J. Pharm, 451, 141 (2002); https://doi.org/10.1016/S0014-2999(02)02225-2.

12. M. Iovu, C. Zalaru, F. Dumitrascu, C.M. Draghici, M. Moraru and E. Cristea, IL Farmaco, 58, 301 (2003); https://doi.org/10.1016/S0014-827X(02)00014-9.

13. F. Manna, F. Chimenti, A. Bolasco, D. Secci, B. Bizzarri, O. Befani, P. Turini, B. Mondovi', S. Alcaro and A. Tafi, Bioorg. Med. Chem. Lett., 12, 3629 (2002); https://doi.org/10.1016/S0960-894X(02)00699-6.

14. X.-D. Yang, J. Chem. Res., 2008, 489 (2008); https://doi.org/10.3184/030823408X340799.

15. A. Verma, V. Kumar, R. Khare and J. Singh, Asian J. Chem., 31, 522 (2019); https://doi.org/10.14233/ajchem.2019.21671.

16. K. Kaur, V. Kumar, V. Beniwal, V. Kumar, K.R. Aneja, V. Sharma and S. Jaglan, Med. Chem. Res., 24, 3863 (2015) https://doi.org/10.1007/s00044-015-1429-2.

17. I. Gulcin, A.Z. Tel and E. Kirecci, Int. J. Food Prop., 11, 450 (2008); https://doi.org/10.1080/10942910701567364.

18. I. Glucin, E. Kirecci, E. Akkemik, F. Topel and O. Hisar, Turk. J. Biol., 34, 175 (2010). 